\section{Regionalização como estratégia para a definição de políticas públicas de controle de homicídios}

\author{
Regionalization as a strategy for the definition \\ of homicide-control public policies
}

1 Departamento de Estatística, Instituto de Ciências Exatas, Universidade Federal de Minas Gerais, Belo Horizonte, Brasil. 2 Centro de Estudos em Criminalidade e Segurança Pública, Universidade Federal de Minas Gerais, Belo Horizonte, Brasil.

Correspondência R. M. Assunção Departamento de Estatística Instituto de Ciências Exatas, Universidade Federal de Minas Gerais. C. P. 702, Belo Horizonte, $M G$ 31.270-901, Brasil. assuncao@est.ufmg.br

\begin{abstract}
This article analyzes the spatial distribution of homicide rates in Minas Gerais State, Brazil, using data from the Ministry of Health Mortality Information System (SIM/MS) from 1996 to 2000. Population data used to calculate rates were also obtained from the Brazilian Ministry of Health. Minas Gerais has a large territory, so the study used a recently developed statistical methodology implemented in the SKATER software to generate homogeneous spatial clusters. The technique obtained 24 spatial clusters, in which municipalities with similar homicide rates were aggregated. Using these results, the authors discuss the possible creation of "public security administration areas" that would allow the implementation of public policies aimed at controlling and diminishing homicides in municipalities with similar characteristics for this type of crime.
\end{abstract}

Regional Health Planning; Violence; Policy Making
Mônica S. Monteiro de Castro 1 Bráulio Figueiredo Alves da Silva 2 Renato M. Assunção 1 Cláudio Chaves Beato Filho 2

O relatório da Organização Mundial da Saúde (OMS) sobre violência ${ }^{1}$ chama a atenção para o grande impacto da violência na saúde da população mundial. Em 2000, cerca de um terço das mortes violentas no mundo foi devido a homicídios (520 mil mortes), e metade foi devida a suicídio (815 mil mortes). Mais de $90 \%$ de todas as mortes relacionadas à violência ocorreram em países de baixa ou média renda. O homicídio representou um problema maior na África e na América, enquanto o suicídio teve maior importância na Europa, no Sudeste Asiático e na região do Pacífico Ocidental. Na América, as taxas de homicídios foram quase três vezes maiores que as taxas de suicídios 1 .

Embora admitindo que a noção de comportamento aceitável é influenciada culturalmente, a OMS define violência como "o uso intencional de força física ou poder, sob a forma de ameaça ou real, contra si mesmo, contra outra pessoa ou contra um grupo ou comunidade, que resulta ou tem uma grande chance de resultar em lesão, morte, dano psicológico, alterações no desenvolvimento ou privações" 2 (p. 5). O relatório defende que a violência é freqüentemente previsível e passível de prevenção, e que os governos devem criar, implementar e monitorar planos para prevenção da violência 1 .

No Brasil, sobretudo nos grandes centros urbanos, os homens com até 30 anos têm sido 
as vítimas mais freqüentes de homicídios. Em estudo recente, Cerqueira \& Lobão ${ }^{3}$, usando dados do Sistema de Informação de Mortalidade do Ministério da Saúde (SIM/MS) para o período de 1981 a 1999, encontraram que as taxas de homicídios entre os homens eram cerca de dez vezes maiores que as taxas para as mulheres. Nesse mesmo trabalho, dados referentes ao ano de 1997 para o Brasil mostraram que cerca de $80 \%$ das vítimas de homicídios possuíam apenas o primeiro grau. Além disso, esses autores verificaram que mais de $50 \%$ dos óbitos de indivíduos entre 10 e 29 anos em São Paulo e no Rio de Janeiro foram homicídios. Um estudo feito, em Belo Horizonte, Minas Gerais 4, sugeriu que grande parte dos homicídios era relacionada ao narcotráfico, mais especificamente a brigas entre gangues rivais disputando o domínio por pontos de venda de drogas ilegais. Esse tipo de padrão de vitimização de homicídios é freqüente em muitos outros centros urbanos no Brasil, nos Estados Unidos e na América Latina.

$\mathrm{O}$ aumento das taxas de criminalidade no Brasil, sobretudo nas grandes cidades, tem motivado a elaboração e implementação de políticas públicas de controle da violência. Políticas públicas voltadas para a diminuição da incidência da criminalidade possuem dois componentes: (a) a definição de tópicos específicos do problema da criminalidade e (b) a definição de estratégias locais de controle.

Com relação ao primeiro componente, para selecionar qual o aspecto do problema da criminalidade a ser abordado, são necessárias informações. Uma fonte de informação bastante utilizada são as estatísticas oficiais. No caso específico dos homicídios, além das estatísticas policiais, podemos levantar informações através de outras bases de dados, como informações do SIM, por exemplo.

Uma outra fonte de dados utilizada são as pesquisas de vitimização, através das quais a experiência da população geral em relação à criminalidade pode sugerir, no nível local ou regional, uma agenda de prioridades pela qual as ações devam ser guiadas. De toda forma, deve-se estar ciente de que qualquer política pública a ser implementada deve ter prioridade política, isto é, o problema que é alvo das ações das políticas públicas deve ser tema presente e relevante na agenda dos tomadores de decisão e dos governantes.

O segundo componente pode envolver, por exemplo, a definição das áreas geográficas relativamente homogêneas de incidência de certos tipos de crimes, agregando municípios em grandes conglomerados, os quais sofreriam os efeitos de políticas públicas focalizadas nos problemas regionais. Nesse sentido, características sócio-econômicas e dos níveis de criminalidade das regiões devem ser levadas em conta no momento da alocação de recursos materiais e humanos.

Procuramos, neste artigo, fornecer subsídios para os dois componentes das políticas ligadas à segurança pública, levantando informações para definição de ações e delimitando locais para sua implementação. Diante do seu efeito sobre a população em geral, seja por causa do elevado número de vítimas ou por causa do medo que esse tipo de crime gera na sociedade, optamos por estudar especificamente os homicídios. Além do mais, em geral, a ocorrência de homicídios está associada a diversos outros delitos de menor gravidade, como furtos, roubos ou assaltos. Ou seja, ao se diminuir o número de homicídios, esses outros delitos também diminuiriam.

A premissa assumida, neste artigo, é de que a implementação de políticas públicas de controle de homicídios baseada em estratégias locais de controle deve levar em consideração as semelhanças e diferenças existentes entre as cidades ou regiões. Ou seja, as propostas de intervenção das políticas públicas devem ser pautadas em diagnósticos que levem em consideração as características específicas de cada região. Não obstante as óbvias implicações de natureza prática, a regionalização de crimes constitui-se também em importante ferramenta para a análise dos correlatos sociais e econômicos de distribuição de crimes. A identificação de padrões espaciais de ocorrência de homicídios leva imediatamente a uma reflexão acerca dos fatores causais responsáveis por essa alocação espacial 5,6,7.

O objetivo deste artigo foi gerar conglomerados espaciais homogêneos com o objetivo de agrupar municípios que sejam semelhantes em relação às suas taxas de homicídios, com vistas a subsidiar uma possível criação de "Núcleos de Gerenciamento em Segurança Pública”, que permitiriam a implementação de políticas públicas de controle de homicídios em grupos de cidades com características semelhantes quanto a esse tipo de crime.

\section{Metodologia}

\section{Dados}

Informações sobre homicídios em Minas Gerais podem ser obtidas de diferentes fontes. Quando um homicídio ocorre, a primeira instituição a ser acionada geralmente é a Polícia Militar, a 
qual registra a ocorrência e coleta algumas informações sobre o contexto no qual o crime ocorreu, sobre o agente, quando esse pode ser identificado, e sobre a vítima, também quando é possível fazer o seu reconhecimento. Em seguida, o Boletim de Ocorrência da Polícia Militar é enviado à Polícia Civil, responsável pela parte investigativa do crime. Essa, por sua vez, encaminha o inquérito ao Ministério Público. Ocasionalmente, os homicídios podem ser notificados diretamente à Polícia Civil, que instaura um inquérito de investigação e dá prosseguimento da mesma forma.

Uma outra fonte de dados sobre homicídios é o SIM, implantado no Brasil desde 1975. O SIM é uma base de informações de atestados de óbitos nacional, da qual se obtém informações sobre homicídios a partir dos registros de mortes ocasionadas por causas externas. Os dados populacionais utilizados para o cálculo das taxas também foram obtidos através do MS. Para este artigo, as informações de óbitos por homicídios para os municípios de Minas Gerais foram obtidas do CD-ROM do SIM, para o período 1996 a 2000. Como em Cruz 8 e em Castro et al. 9, o filtro utilizado para a recuperação dos óbitos foram os códigos X85 a Y09 da Classificação Internacional de Doenças - Décima Revisão (CID-10) 10, que correspondem às categorias habitualmente classificadas como homicídios, e os códigos Y22 a Y24 e Y28 e Y29 da CID-10, que correspondem a categorias de morte de intenção não determinada causada por arma de fogo ou arma branca.

\section{Análise estatística}

Para estimar o risco de ocorrência de um evento em pequenas áreas, existem alguns métodos de estimação. O mais simples e usual é a taxa bruta, que consiste na razão entre o número de eventos ocorridos numa área em um período de tempo e o número de pessoas-ano expostas à ocorrência desse evento na mesma área e período. Uma alternativa a esse método são os Estimadores Bayesianos Empíricos 11,12. "Esta é uma das melhores estimativas disponíveis para estimar riscos em pequenas áreas" 13 (p. 135).

O Estimador Bayesiano Empírico permite que a informação a respeito do risco contida nos dados de todas as outras áreas seja usada para estimar o risco de determinada área. $\mathrm{O}$ método elimina parcialmente a variabilidade presente nas taxas brutas que não estão associadas a fatores de risco. Mais especificamente, o Estimador Bayesiano Empírico é uma taxa ajustada da forma

$$
b_{i}=w_{i} t_{i}+\left(1-w_{i}\right) m
$$

na qual $t_{i}$ é a taxa de homicídios na área, $m$ é a taxa global estadual e $w_{i}$ é um peso entre 0 e 1 que depende do tamanho da população da área $i$. Quanto maior a área $i$, mais próximo de 10 peso $w_{i}$. Assim, municípios com grande população possuem a estimativa bayesiana empírica $b_{i}$ muito próxima da taxa $t_{i}$ daqueles municípios. Já para os municípios com população pequena, o valor de $b_{i}$ será intermediário entre $t_{i}$ e $m$.

O mapa das taxas bayesianas $b_{i}$ encontrase na Figura 1. Nesse mapa e no restante deste trabalho, usamos a divisão de Minas Gerais em 853 municípios, divisão político-administrativa vigente a partir de 1995. Embora seja possível identificar certas concentrações de áreas com valores $b_{i}$ similares, mesmo usando as taxas bayesianas, o mapa não apresenta regiões nitidamente diferenciadas. É comum encontrarmos valores bastante discrepantes entre áreas vizinhas. Com base apenas nesse mapa, torna-se difícil agregar áreas sem usar uma dose excessiva de arbitrariedade e subjetividade.

Diante desse problema, escolhemos uma metodologia estatística que possibilita a criação de conglomerados espaciais a partir de variáveis de interesse. As regionalizações foram feitas levando em conta critérios de homogeneidade e restrições de contigüidade, através de métodos baseados em árvores de regressão e em árvores geradoras mínimas (Assunção RM, 2000. Avaliação do uso de conglomerados espaciais para a definição das áreas de ponderação do censo demográfico 2000. Assunção RM, 2001. Análise de conglomerados espaciais. Relatórios finais de pesquisas apresentados à Fundação Instituto Brasileiro de Geografia e Estatística) e implementados no programa SKATER 14, disponível gratuitamente no endereço eletrônico http://www.est.ufmg.br/leste.

Existem outras possibilidades metodológicas propostas na literatura para formação de conglomerados 15,16,17,18,19,20, mas não existe nenhuma comparação sistemática entre elas. Optamos então por trabalhar com um método cuja implementação está disponível em programa gratuito e cuja aplicação em dados de saúde e violência é inédita na literatura.

O método de criação de conglomerados utilizado pelo SKATER consiste na geração de conglomerados espaciais compostos internamente de municípios muito similares com respeito à variável que se deseja analisar. Ao mesmo tempo, municípios de conglomerados diferentes são distintos entre si o máximo possível.

Assim, o mapa do Estado de Minas Gerais foi representado por um grafo (Figura 2), no qual 


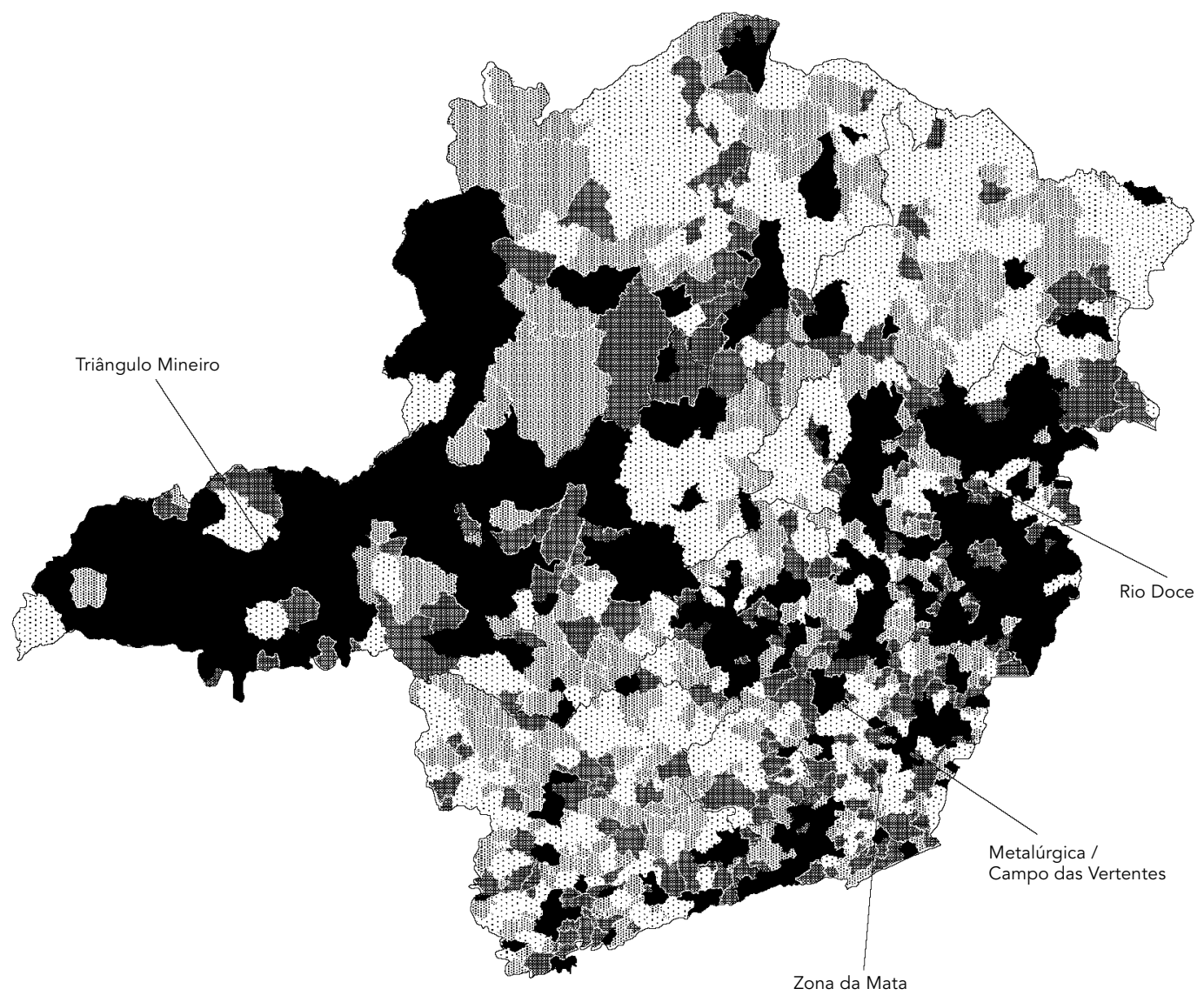

Taxa Bayesiana de homicídios por 100 mil pessoas Minas Gerais - 1996 a 2000

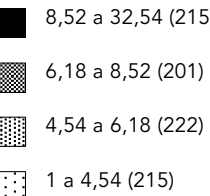

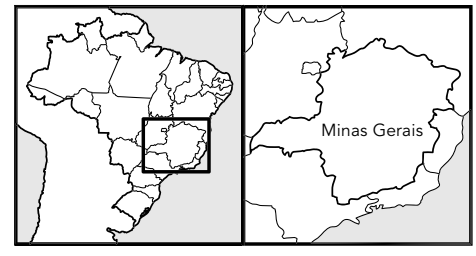

cada município corresponde a um nó e no qual municípios vizinhos são ligados por uma aresta. Dois municípios são considerados vizinhos se eles compartilham limites geográficos. O grau de dissimilaridade entre dois municípios vizinhos $i$ e $j$ é definido como a diferença elevada ao quadrado entre suas taxas bayesianas empíricas $\left[\left(b_{i}-b_{j}\right)^{2}\right]$.

Em seguida, o método procura simplificar o grafo apagando arestas de forma a ficar com um grafo reduzido, porém ainda totalmente conexo. Isto é, no grafo reduzido, é possível ir de uma área a qualquer outra no mapa seguindo um caminho sucessivo de arestas. As arestas a serem apagadas são aquelas que conectam municípios muito dissimilares. Assim, ao passar de uma área a outra, teremos, em geral, uma diferença pequena nas taxas das áreas envolvidas nessa passagem. Por fim, o método irá gerar uma árvore tal que, se for apagada qualquer aresta adicional, o grafo ficará dividido em dois grafos desconectados, os quais serão 


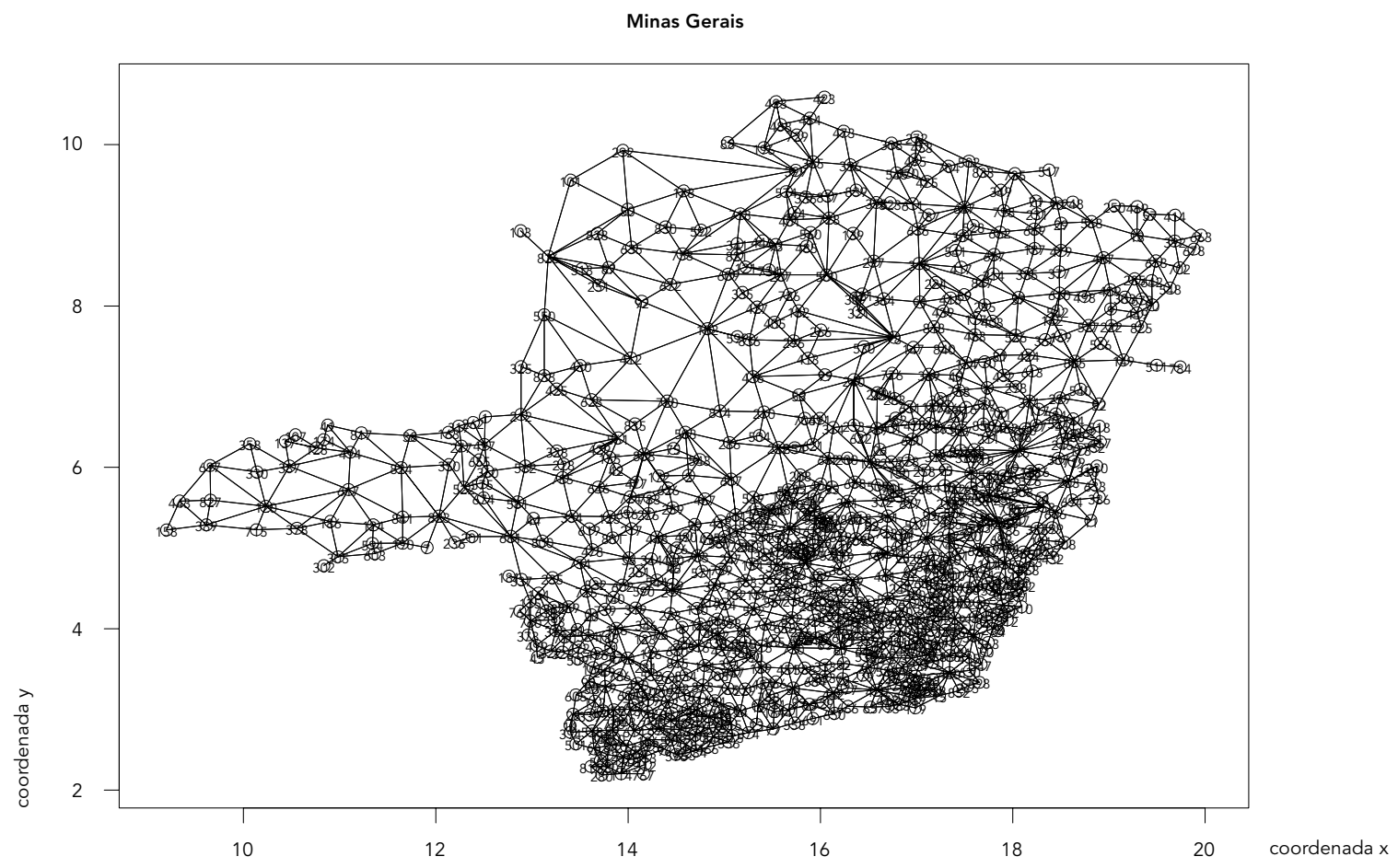

candidatos a constituírem dois conglomerados espaciais. Dessa forma, obtemos a Árvore Geradora Mínima (Figura 3), que possui a menor soma dos graus de dissimilaridade entre suas arestas.

A partir da Árvore Geradora Mínima, o procedimento para particionar o mapa e obter conglomerados espaciais é bastante simplificado: basta percorrer todas as arestas que compõem a Árvore Geradora Mínima e apagar aquela que cria os dois conglomerados espaciais $G_{1}$ e $G_{2}$ com o menor desvio $D$, definido como

$$
D=\sum_{i \in G_{1}}\left(b_{i}-m_{1}\right)^{2}+\sum_{i \in G_{2}}\left(b_{i}-m_{2}\right)^{2}
$$

no qual $m_{1}$ e $m_{2}$ são as taxas médias bayesianas dentro de $G_{1}$ e $G_{2}$, respectivamente. Fazendo isso, obtêm-se como resultado dois grafos desconectados, que podem ser vistos como dois conglomerados espaciais. O grau de dissimilaridade do mapa partido em dois conglomerados é a soma das dissimilaridades das arestas não apagadas nos dois grafos formados. A seguir, o método particiona um dos conglomerados formados, $G_{1}$ e $G_{2}$, apagando aquela aresta que cria três conglomerados com o menor desvio interno. Se esse processo for repetido, ele terminará quando todas as arestas forem apagadas, gerando tantos conglomerados quantos forem os municípios que compõem o Estado. Mas o que se deseja é que o método possibilite a geração de conglomerados espaciais de municípios que sejam o máximo possível homogêneos entre si, de forma que esses conglomerados sejam compostos por um certo número de municípios semelhantes quanto às taxas de homicídios. Para isso, o método permite analisar o melhor momento para interromper a poda da Árvore Geradora Mínima, através da análise da diminuição do desvio interno (Figura 4), à medida que mais conglomerados vão sendo gerados. Existe uma diminuição rápida no início, quando o número de conglomerados é pequeno. A partir de um certo ponto, essa diminuição é pequena, significando que a geração de mais conglomerados não aumenta substancialmente a homogeneidade interna dos conglomerados. Para este artigo, as funções do SKATER foram ligeiramente modificadas para permitir a limitação simultânea do número de 
Figura 3

Árvore geradora mínima dos municípios de Minas Gerais, Brasil, gerada a partir da remoção de arestas do grafo de vizinhança da Figura 2.

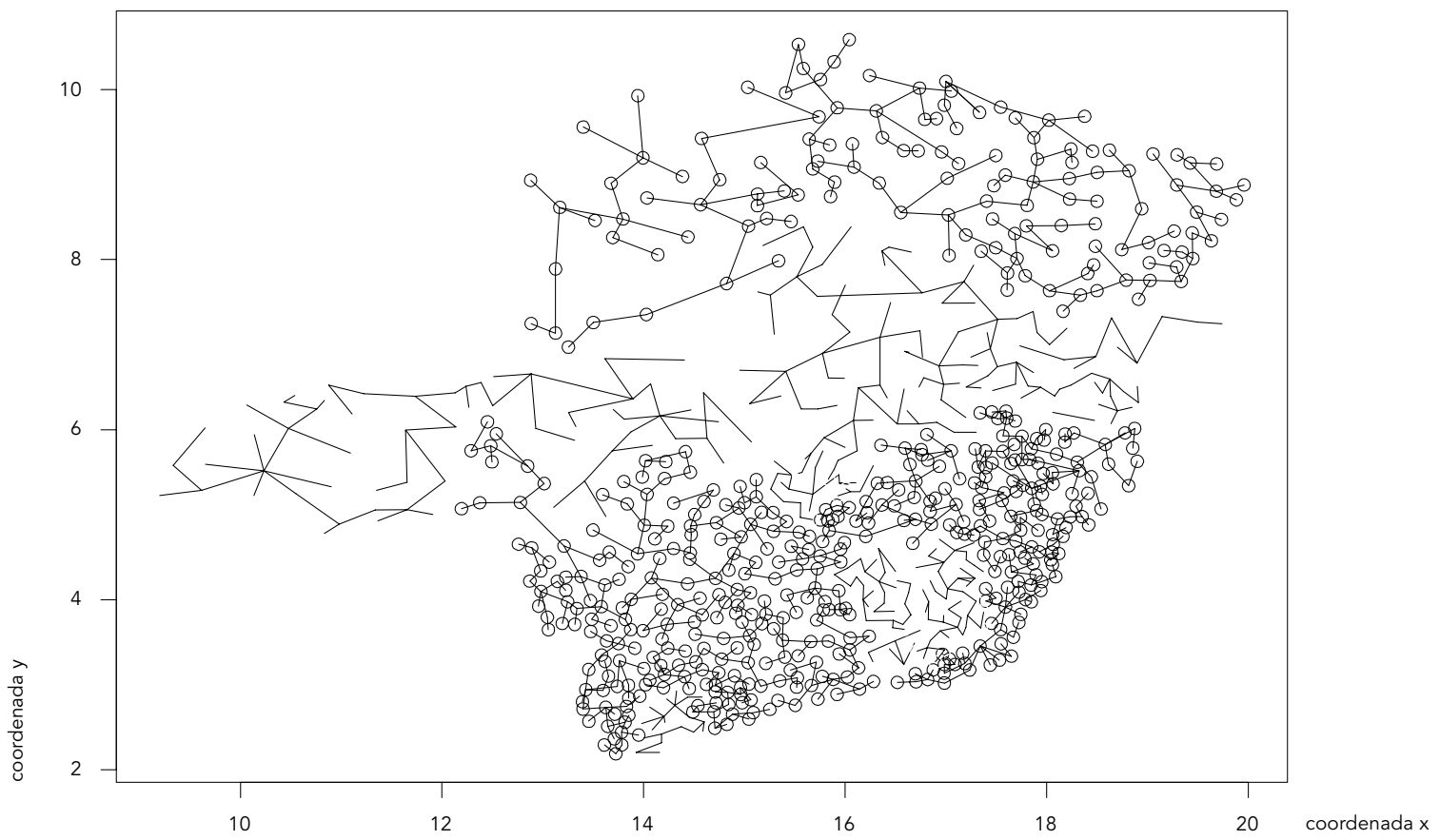

Figura 4

Queda dos desvios durante o processo de partição dos conglomerados



conglomerados e da população mínima em cada conglomerado, no caso, 250 mil pessoas.

\section{Resultados}

Analisando a série histórica de homicídios em Minas Gerais, verifica-se que, de 1980 até 1988, a taxa de homicídio no Estado caiu ligeiramente ano após ano, com um pequeno pico registrado em 1984. A taxa de homicídio em 1980 foi de 8,7 por 100 mil pessoas e caiu para 6,7 por 100 mil em 1988. De 1989 até 1994, houve um pequeno crescimento das taxas em relação ao período anterior. A partir de 1995, começa a ocorrer uma forte tendência de crescimento das taxas, que se acentua principalmente nos dois últimos anos do período, alcançando 11,5 homicídios por 100 mil pessoas em 2000.

A Figura 1 apresenta a distribuição espacial das taxas de homicídios dos municípios mineiros, sobreposta à divisão do Estado em macrorregiões administrativas. Tomando-se os $25 \%$ dos municípios com as maiores taxas, verificamos que eles estão concentrados praticamente em 
quatro grandes regiões do Estado: Triângulo Mineiro, Região Metalúrgica/Campo das Vertentes (onde está localizada a região Metropolitana), Rio Doce e Zona da Mata, mais especificamente os municípios circunvizinhos a Juiz de Fora. O restante do Estado apresenta taxas menos elevadas.

Foram selecionados quatro momentos no procedimento de partição para criar conglomerados espaciais: sete, onze, dezesseis e vinte e quatro conglomerados (Figura 4). A partir de 24 conglomerados, não existem mais motivos técnicos para fazer novas divisões, pois a queda subseqüente dos desvios é muito pequena. Isto é, os conglomerados criados já são suficientemente homogêneos e não mais necessitam de partições adicionais.

Os conglomerados criados sucessivamente podem ser vistos na Figura 5. A Figura 5a representa o Estado dividido em sete conglomerados espaciais formados em função da homogeneidade de homicídios. A partir da legenda, podemos identificar as taxas médias das regiões e o número de municípios que compõem cada conglomerado (entre parênteses). A taxa média é a razão entre o número total de homicídios no período de 1996 a 2000 na região e a população total dessa região. Como os conglomerados formados possuem grande população, não foi necessário adotar estimativas bayesianas.

Nessa primeira divisão, podemos verificar que mais de $65 \%$ dos municípios são agrupados em apenas um único conglomerado, cuja taxa é de 5,2 homicídios por $100 \mathrm{mil}$ pessoas. Adicionalmente, notamos a presença de pequenos conglomerados, como o caso da região de Governador Valadares (dois municípios no Leste do Estado) e região Metropolitana (cinco municípios no Centro-Sul do Estado). Isso se deve ao fato de que essas são áreas onde a ocorrência de homicídios é extremamente elevada, principalmente no caso da cidade de Governador Valadares, que tem uma pequena população, se comparada aos grandes centros urbanos, mas cuja incidência de homicídios é muito elevada, de 32,4 homicídios por 100 mil pessoas.

A segunda partição foi de 11 conglomerados, como pode ser vista na Figura 5b. A grande área que agregava mais de 65\% dos municípios dividiu-se em outros conglomerados de menor tamanho. Esse processo adicional de partição de conglomerados dividiu apenas o conglomerado com o maior território. Isso se deu porque esse grande conglomerado ainda apresenta uma grande heterogeneidade, se comparada com aquela existente nos outros seis conglomerados menores da Figura 5a.

Continuando o procedimento de partição de conglomerados espaciais, produziu-se a re- gionalização em 16 conglomerados (Figura 5c). É importante notar que todos conglomerados espaciais formados na primeira divisão, exceto o maior deles, são mantidos inalterados nas divisões sucessivas. Isso significa que essas regiões são muito homogêneas em relação ao conglomerado com maior território, ou seja, elas se destacam como sub-regiões bastante distintas das demais e com altas taxas de homicídios.

Finalmente, a Figura 5d apresenta os 24 conglomerados de municípios mineiros, configuração considerada a melhor regionalização obtida pelo método. Pode-se verificar que as taxas de homicídios entre os conglomerados formados apresentam uma grande variação, sendo que, em alguns casos, temos regiões com 2,1 homicídios por 100 mil habitantes e, no outro extremo, naquelas regiões já identificadas com altas taxas de homicídios, temos valores que alcançam 32,4 homicídios por 100 mil habitantes.

\section{Discussão}

O resultado da regionalização de Minas Gerais segundo taxas de homicídios sugere algumas hipóteses sobre a distribuição espacial dos homicídios no Estado. Com relação ao Triângulo Mineiro, as elevadas taxas de homicídios podem estar associadas ao grande número de crimes contra o patrimônio que caracterizam os seus municípios. Segundo o Atlas da Criminalidade de Minas Gerais 21 e também segundo dados recentes da Polícia Militar de Minas Gerais (PM-MG), a região do Triângulo Mineiro teve as maiores taxas estaduais de roubos e assaltos no período analisado neste artigo. Além disso, aproximadamente $60 \%$ dos crimes foram cometidos com uso de armas de fogo. Isso sugere que altas taxas de homicídios nessa região podem ser conseqüência de uma elevada incidência de crimes contra o patrimônio, nos quais foram utilizadas armas de fogo.

Com relação à Região Metropolitana de Belo Horizonte (RM-BH), pode-se sugerir que as elevadas taxas de homicídios estejam associadas ao tamanho das cidades, ou melhor, à alta densidade populacional na região, principalmente em algumas áreas de Belo Horizonte, Betim e Contagem. Dentro dessas cidades, existem bolsões de pobreza cujo ambiente de desorganização social proporciona um contexto no qual o conflito entre pessoas é estimulado 22 . Ao mesmo tempo, esses centros urbanos têm se caracterizado, nos últimos anos, por uma elevada ocorrência de crimes relacionados ao tráfico de drogas, com grande quantidade de 
Figura 5

Divisão de Minas Gerais, Brasil, em 7, 11, 16 e 24 conglomerados espaciais de homicídios

Figura $5 a$
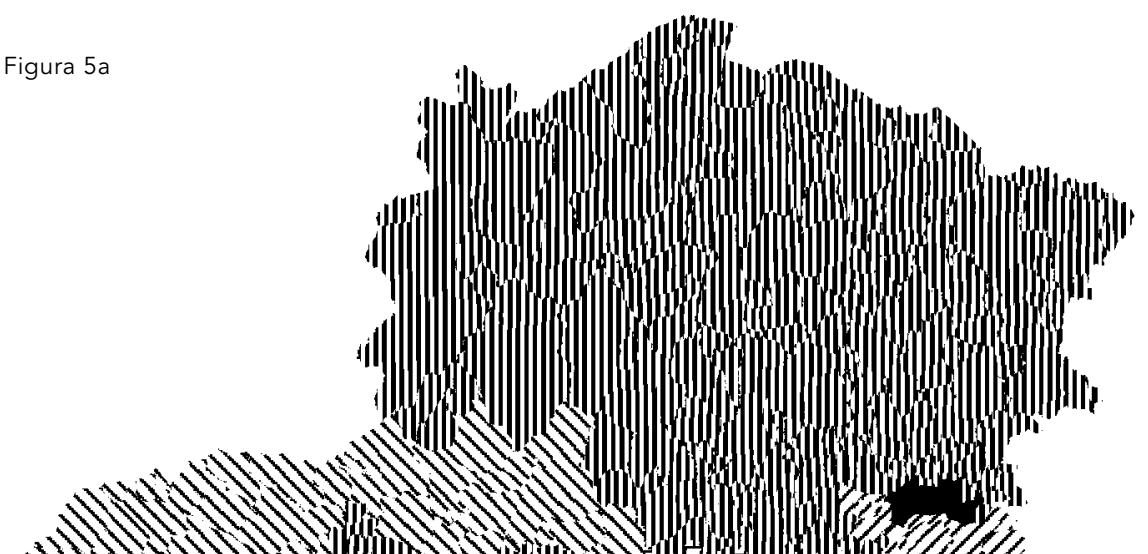

Conglomerados de homicídios Minas Gerais - 1996 a 2000

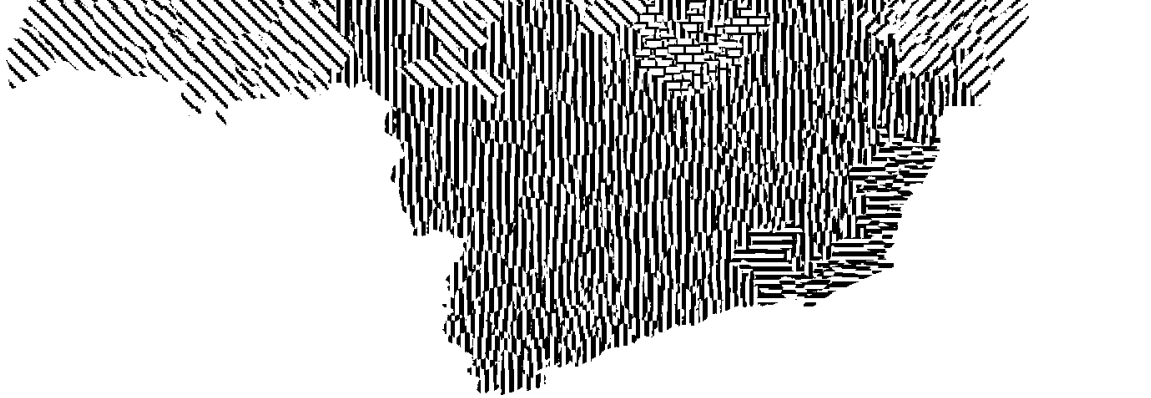

II $5,24(670)$

E $8,47(50)$

10,36 (40)

$11,26(63)$

巴 12,04 (23)

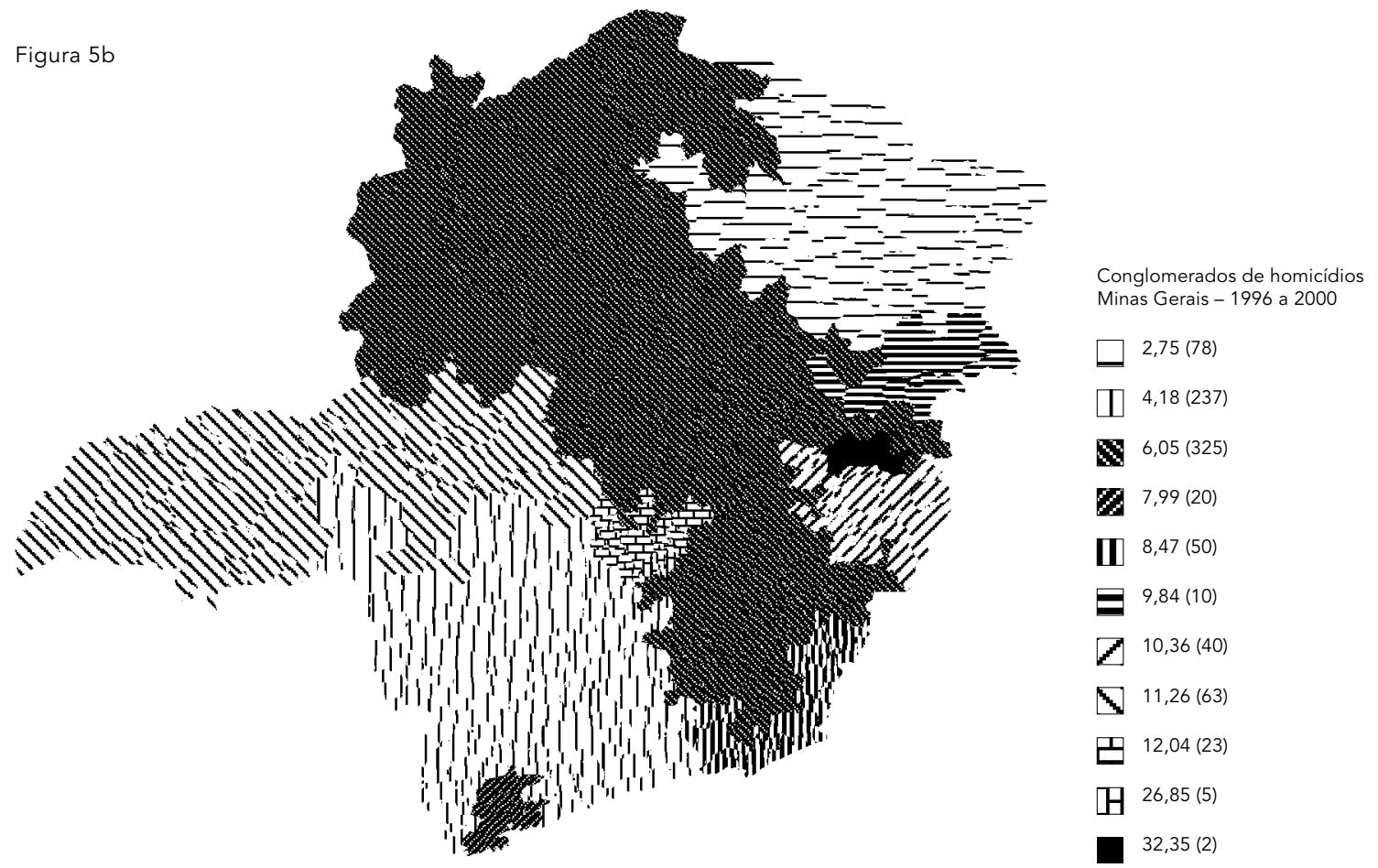


Figura 5 (continuação)

Divisão de Minas Gerais, Brasil, em 7, 11, 16 e 24 conglomerados espaciais de homicídios.
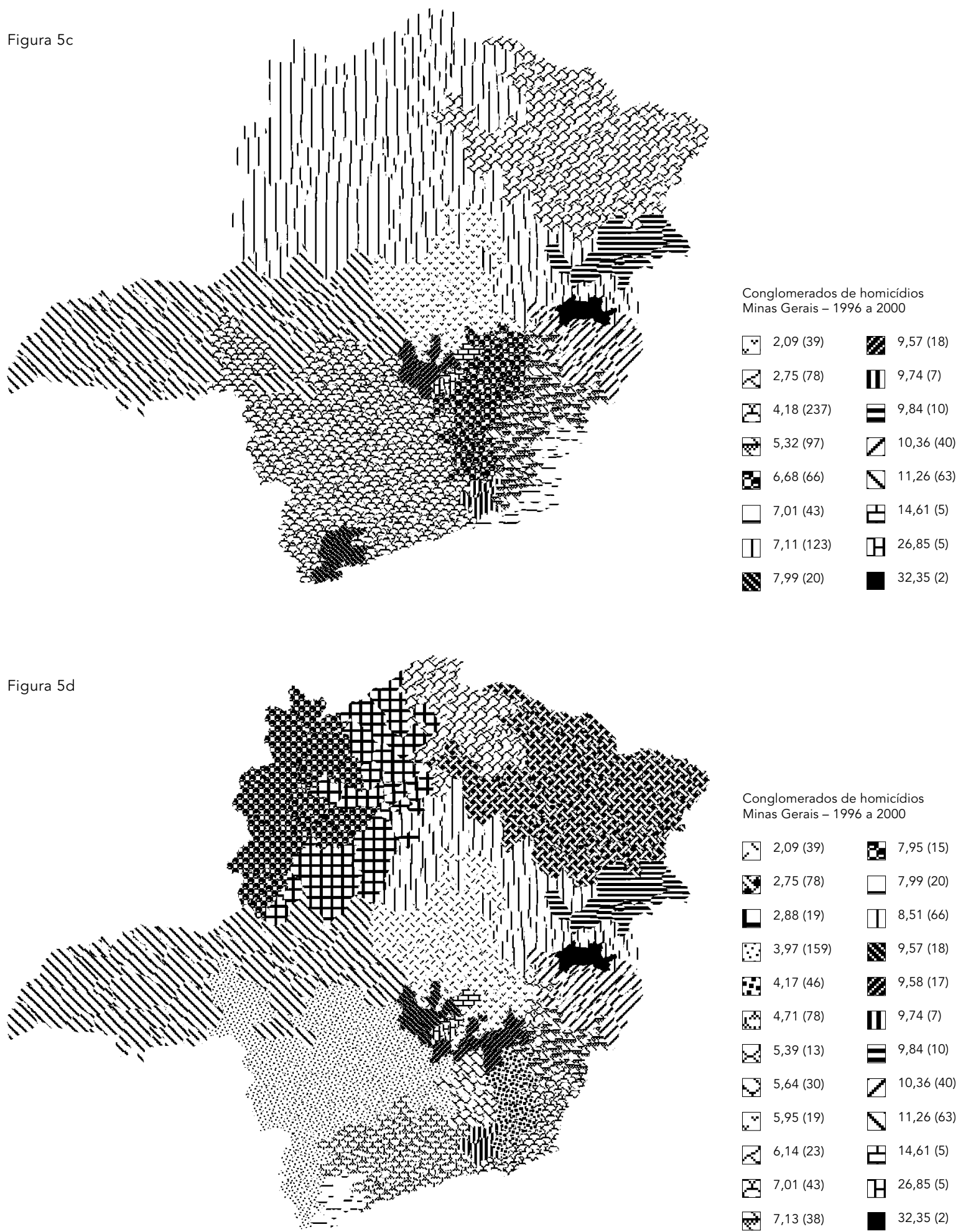

Conglomerados de homicídios Minas Gerais - 1996 a 2000

$\begin{array}{ll}\mathbf{D}^{2,09(39)} & \mathbf{Q}^{7,95(15)} \\ \mathbf{D}^{2,85(78)} & \square^{7,99(20)} \\ \mathbf{D}^{3,51(66)}\end{array}$

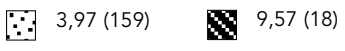

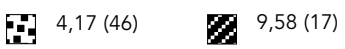

内. $4,71(78)$ || $9,74(7)$

过, 59 (13) $9,84(10)$

$\square^{5,64(30) \quad 7^{10,36(40)}}$

$5,95(19) \quad 11,26(63)$

$\triangle^{6,14(23)}$ ${ }^{14,61(5)}$

7.01(43) [प $26,85(5)$

7,13(38) \32,35(2) 
drogas apreendidas. Dados da Polícia Civil de Minas Gerais, citados no Relatório sobre a Apreensão de Drogas em Minas Gerais, em 1999 e 2000, destacam a 52a Delegacia Regional de Segurança Pública, responsável pela RM-BH, como a delegacia que apresenta os maiores registros desse tipo de crime. Estudos sugerem que homicídios nos grandes centros urbanos são fortemente associados a brigas por pontos de venda e comércio ilegal de drogas e por acertos de contas entre gangues rivais 4 .

Na região do Vale do Rio Doce, as cidades de Governador Valadares, Teófilo Otoni e Malacacheta têm elevadas taxas de homicídios. Uma das causas desse achado pode ser a alta freqüência de resolução de conflitos entre pessoas com o uso de arma de fogo que ocorre nessas regiões 21, o que acaba resultando em óbitos.

Finalmente, para a Zona da Mata, a proximidade do Estado do Rio de Janeiro pode justificar as elevadas taxas de homicídios encontradas em alguns dos maiores municípios que a compõem, como Juiz de Fora e Santos Dumont. Essa não é uma região tipicamente violenta se comparada às outras do Estado, e as taxas de crimes de seus municípios apresentam uma relativa estabilidade, segundo dados da PM-MG. Entretanto, no período de 1996 a 2000, já se pode verificar o crescimento dos assaltos com utilização de arma de fogo, o que poderia ter associação com os homicídios registrados, como no caso do Triângulo Mineiro.

Ao ser tratada como um problema de saúde pública e de segurança pública, a questão dos homicídios torna-se objeto de políticas públicas orientadas para a redução dos seus efeitos indesejáveis sobre indivíduos, coletividades e sobre a própria sociedade. Nesse caso, deve-se considerar que os "programas devem pautar-se por metas muito claras e definidas a serem alcançadas; que a condição desejável a ser perseguida pode ser a redução de alguns tipos específicos de crime a um custo razoável para sua implementação" 23 (p. 340).

No que diz respeito a uma proposta efetiva de controle de homicídios de modo regionalizado, como mencionado neste artigo, temos, como referência, um plano de intervenção implementado em Belo Horizonte, o Fica Vivo 24. Esse programa contou com a parceria de diversas instituições, tais como as Polícias Militar e Civil de Minas Gerais, Polícia Federal, Ministério Público, Prefeitura de Belo Horizonte, Universidade Federal de Minas Gerais, Serviço Brasileiro de Apoio às Micro e Pequenas Empresas (SEBRAE), Câmara de Dirigentes Lojistas, organizações não-governamentais, movimentos sociais e comunidade local. O programa con- siste no desenvolvimento de estratégias eficazes de intervenção, através do uso da metodologia de solução de problemas, a fim de reduzir a incidência de homicídios.

A metodologia de solução de problemas é uma estratégia localizada que possui quatro etapas. A primeira delas, identificação, tem como objetivo descobrir os problemas associados aos homicídios, selecionar prioridades de ação e definir responsabilidades. A segunda fase, chamada de análise, consiste na compreensão mais profunda do problema, através do seu estudo de forma detalhada. Nesse caso, procura-se conhecer minuciosamente o contexto no qual o crime ocorre, o envolvimento das vítimas e agentes, e sua distribuição espacial e temporal. Na terceira fase, denominada respos$t a$, tem-se a intervenção propriamente dita. Isto é, seleciona-se uma solução ou plano de ação estratégico, que é colocado em prática. Finalmente, tem-se a fase de avaliação, na qual se procura criar critérios objetivos para avaliação do funcionamento e da efetividade do projeto.

Os resultados obtidos a partir da implementação do Programa Fica Vivo foram muito satisfatórios e indicaram uma grande capacidade de resposta ao problema dos homicídios. Em apenas seis meses de implementação, o aglomerado do Morro das Pedras sofreu uma redução de $47 \%$ das taxas de homicídios, se comparado aos seis meses anteriores à implementação do projeto na mesma região. Ao mesmo tempo, quando se analisaram outras áreas da cidade reconhecidamente violentas e que não receberam a intervenção, percebeu-se um crescimento de $12 \%$ nas taxas de homicídios. É importante observar que o programa não se apresentou eficaz apenas na diminuição dos homicídios na área, mas foi importante também para a diminuição de outros crimes violentos no mesmo período, como tentativa de homicídio, assaltos e roubos a padarias, supermercados, ônibus e táxis.

De modo análogo, a regionalização de Minas Gerais em áreas homogêneas de homicídios indica diversas possibilidades de ação no sentido de explorar mais detalhadamente os fatores associados à ocorrência de homicídios nos municípios que compõem cada região. Através da metodologia utilizada, foi possível regionalizar Minas Gerais e levantar a possibilidade da criação, em cada conglomerado espacial, de "Núcleos de Gerenciamento em Segurança Pública”, compostos por lideranças municipais, agências policiais, Ministério Público e sociedade civil, a fim de identificar, analisar e responder ao problema específico de cada região. 


\section{Resumo}

Neste artigo, analisamos a distribuição espacial das taxas de homicídios no Estado de Minas Gerais, Brasil, utilizando dados do Sistema de Informações de Mortalidade do Ministério da Saúde (SIM-MS), no período de 1996 a 2000. Os dados populacionais utilizados para o cálculo das taxas também foram obtidos através do MS. Diante da grande extensão territorial do Estado, utilizamos uma metodologia estatística desenvolvida recentemente, implementada no programa SKATER, para a geração de conglomerados espaciais homogêneos. Como resultado, obtivemos 24 conglomerados espaciais, nos quais os municipios semelhantes em relação às taxas de homicídios ficaram agrupados. A partir desses resultados, discutimos a possibilidade da criação de "Núcleos de Gerenciamento em Segurança Pública", que permitiriam a implementação de políticas públicas voltadas para o controle e a diminuição de homicídios em cidades com características semelhantes no que diz respeito a esse tipo de crime.

Regionalização; Violência; Formulação de Políticas

\section{Colaboradores}

Os dois primeiros autores em conjunto desenharam o estudo e escolheram os métodos de análise. R. M. Assunção elaborou o método estatístico e desenhou o programa SKATER usado na análise. B. F. A. Silva preparou os dados e rodou o programa SKATER. Uma primeira versão do artigo foi escrita pelos dois primeiros autores. Todos os quatro autores interpretaram os resultados e escreveram a versão final do artigo.

\section{Referências}

1. Krug EG, Dahlberg LL, Mercy JA, Zwi A, Lozano R. World health report on violence and health. Geneva: World Health Organization; 2002.

2. World Health Organization. Global consultation on violence and health. Violence as a public health priority. Geneva: World Health Organization; 1996.

3. Cerqueira D, Lobão W. Condicionantes sociais, poder de polícia e o setor de produção criminal. Rio de Janeiro: Instituto de Pesquisa Econômica Aplicada; 2003.

4. Beato Filho CC, Assunção RM, Silva BFA, Marinho FC, Almeida MCM. Conglomerados de homicídios e o tráfico de drogas em Belo Horizonte, 1995 a 1999. Cad Saúde Pública 2001; 17:1163-71.

5. Shaw C, Henry M. Juvenile delinquency and urban areas. Chicago: University of Chicago Press; 1942.

6. Sampson RJ, Raudenbush SW. Systematic social observation of public spaces: a new look at disorder in urban neighborhoods. AJS 1999; 105:603-51.

7. Bursik Jr. RJ, Grasmick H. Neighborhoods and crime: the dimensions of effective community control. New York: Lexington; 1993.

8. Cruz O. Homicídios no Estado do Rio de Janeiro: análise da distribuição espacial e sua evolução [Dissertação de Mestrado]. São Paulo: Faculdade de Saúde Pública, Universidade de São Paulo; 1996.

9. Castro MSM, Assunção RM, Durante MO. Comparação de dados sobre homicídios entre dois sistemas de informação, Minas Gerais. Rev Saúde Pública 2003; 37:168-76.

10. Organização Mundial da Saúde. Classificação internacional de doenças e problemas relacionados à saúde: décima revisão. São Paulo: Centro Colaborador da OMS para Classificação de Doenças em Português; 1993.

11. Marshall RJ. Mapping disease rates and mortality rates using empirical Bayes estimators. Appl Stat 1991; 40:283-94.

12. Assunção RM, Reis EA, Marchesini PB, Sawyer DO. Mapas de malária em Rondônia usando o Estimador Bayesiano Empírico para dados binários. Rev Bras Estat 1999; 60:69-94.

13. Esteve J, Benhamou E, Raymond L. Statistical methods in cancer research. v. IV - Descriptive epidemiology. Lyon: International Agency for Research on Cancer/World Health Organization; 1994.

14. Assunção RM, Lage JP, Reis EA. Análise de conglomerados espaciais via árvore geradora mínima. Rev Bras Estat; no prelo.

15. Haining R. Classifying space and analyzing the consequences: spatial analysis of health data. In: Gaul W, Pfeifer D, editors. From data to knowledge. Berlin: Springer-Verlag; 1996. p. 45-57.

16. Carvalho MS, Cruz OG, Nobre FF. The use of multivariate cluster analysis and aggregation algorithm to propose a new spatial partitioning of Rio de Janeiro, Brazil. Stat Med 1996; 15:1885-94.

17. Carvalho MS, Cruz OG, Nobre FF. Fuzzy classification applied to modeling socioeconomic profiles for spatial health analysis. Proceedings of the EIS'98 - International ICSC Symposium on Engi- 
neering of Intelligent Systems. [CD-ROM]. February 11-13 1998. Tenerife: University of La Laguna; 1998.

18. Osnes K. Interative random aggregation of small units using regional measures of spatial auto-correlation for cluster localization. Stat Med 1999; 18:707-25.

19. Rezende FS, Almeida RV, Nobre FF. Diagramas de Voronoi para a definição de áreas de abrangência de hospitais públicos no Município do Rio de Janeiro. Cad Saúde Pública 2000; 16:467-75.

20. Kiang M. Extending the Kohonen self-organizing map networks for clustering analysis. Computational Statistics and Data Analysis 2001; 38:16180.

21. Fundação João Pinheiro/Polícia Militar de Minas Gerais/Universidade Federal de Minas Gerais. Criminalidade Violenta em Minas Gerais, de 1986 a 1997. [CR-ROM]. Belo Horizonte: Fundação João Pinheiro/Polícia Militar de Minas Gerais/ Universidade Federal de Minas Gerais; 1998.
22. Sampson RJ, Groves WB. Community structure and crime: testing social-disorganization theory. AJS 1989; 94:774-802.

23. Beato Filho CC. Políticas públicas de segurança e a questão policial: eficiência, eqüidade e accountability. In: Melo MA, organizador. Reforma do Estado e mudança institucional no Brasil. Recife: Massangana; 1999. p. 335-65.

24. Beato Filho CC, Souza RSR, Durante MO, Silva BFA, Silveira AM. Programa Fica Vivo: ações simples, resultados efetivos. Informativo do Centro de Estudos de Criminalidade e Segurança Pública. http://www.crisp.ufmg.br (acessado em Fev/2003).

Recebido em 23/Out/2003

Versão final reapresentada em 05/Abr/2004

Aprovado em 30/Abr/2004 